\title{
Cumulative damage effect of jaundice may be an effective predictor of complications in patients undergoing radical resection of Bismuth type II or above hilar cholangiocarcinoma
}

\author{
Le Luo $^{1,2 \#}$, Yutong Yao ${ }^{2 \#}$, Haotian Liao ${ }^{1 \#}$, Jiwei Huang ${ }^{1}$, Mingheng Liao ${ }^{1}$, Jinju Wang ${ }^{1}$, Kefei Yuan ${ }^{1}$, \\ Yong Zeng ${ }^{1}$
}

${ }^{1}$ Department of Liver Surgery \& Liver Transplantation, State Key Laboratory of Biotherapy and Cancer Center, West China Hospital, Sichuan University and Collaborative Innovation Center of Biotherapy, Chengdu, China; ${ }^{2}$ Department of Hepatobiliary and Pancreatic Surgery Center, Cell Transplantation Center, Sichuan Provincial People's Hospital, University of Electronic Science and Technology of China, Chengdu, China

Contributions: (I) Conception and design: L Luo, Y Yao; (II) Administrative support: K Yuan, Y Zeng; (III) Provision of study materials or patients: H Liao, J Huang, M Liao; (IV) Collection and assembly of data: L Luo, Y Yao, M Liao, J Wang; (V) Data analysis and interpretation: L Luo, Y Yao, H Liao; (VI) Manuscript writing: All authors; (VII) Final approval of manuscript: All authors.

"These authors contributed equally to this work.

Correspondence to: Yong Zeng; Kefei Yuan. State Key Laboratory of Biotherapy and Cancer Center, West China Hospital, Sichuan University and Collaborative Innovation Center of Biotherapy, Chengdu, China. Email: zengyong2021@126.com; ykf13@163.com.

Background: There is currently no preoperative risk assessment system for predicting complications after radical resection of hilar cholangiocarcinoma. This study examined the association between the cumulative damage effect of jaundice (CDEJ) and the complications of radical resection of Bismuth II or above hilar cholangiocarcinoma.

Methods: Patients who underwent radical resection of hilar cholangiocarcinoma at the Department of Hepatobiliary Surgery, West China Hospital of Sichuan University, from April 2010 to January 2018 were retrospectively included.

Results: Of the 171 included patients, 115 (67.3\%) patients experienced complications. Multivariate analysis found that CDEJ [odds ratio $(\mathrm{OR})=1.0001,95 \%$ confidence interval $(95 \% \mathrm{CI})=1.000027-1.000239$, $\mathrm{P}=0.014]$, cholangitis $(\mathrm{OR}=9.638,95 \% \mathrm{CI}=2.683-34.622, \mathrm{P}=0.001)$, and preoperative bilirubin $(\mathrm{OR}=1.006$, $95 \% \mathrm{CI}=1.002-1.01, \mathrm{P}=0.004)$ were independently associated with the incidence of complications. CDEJ $(\mathrm{OR}=1.0001,95 \% \mathrm{CI}=1.00001-1.00019, \mathrm{P}=0.024)$, age $(\mathrm{OR}=1.083,95 \% \mathrm{CI}=1.029-1.14, \mathrm{P}=0.002)$, preoperative bilirubin $(\mathrm{OR}=1.083,95 \% \mathrm{CI}=1.029-1.14, \mathrm{P}=0.002)$, and future liver remnant (FLR) ( $\mathrm{OR}=0.963,95 \% \mathrm{CI}=0.941-0.986, \mathrm{P}=0.002)$ were independently associated with hepatic failure. To predict the incidence of complications, the following criteria were used. For the CDEJ cutoff of 2,151, the area under the receiver operating characteristic curve (AUC) was 0.69 (95\% CI $=0.615-0.759)$, the sensitivity was $66.09 \%$, and the specificity was $69.64 \%$. For the preoperative bilirubin cutoff of $111.7 \mu \mathrm{mol} / \mathrm{L}$, the AUC was 0.65 (95\% CI $=0.573-0.721)$, the sensitivity was $84.35 \%$, and the specificity was $42.86 \%$. To predict hepatic failure, the following criteria were used. For the CDEJ cutoff of 3,931.95, the AUC was 0.605 (95\% $\mathrm{CI}=0.582-0.679)$, the sensitivity was $51.28 \%$, and the specificity was $70.45 \%$. For the preoperative bilirubin cutoff of $115.9 \mu \mathrm{mol} / \mathrm{L}$, the AUC was 0.638 (95\% CI $=0.561-0.71)$, the sensitivity was $92.31 \%$, and the specificity was $32.58 \%$. For the FLR cutoff of 50, the AUC was 0.638 (95\% CI $=0.515-0.667)$, the sensitivity was $48.72 \%$, and the specificity was $78.79 \%$.

Conclusions: CDEJ was independently associated with complications and can moderately predict complications after surgical resection of hilar cholangiocarcinoma.

Keywords: Klatskin tumor; jaundice; obstructive; postoperative complications; risk assessment 
Submitted Mar 18, 2021. Accepted for publication May 06, 2021.

doi: $10.21037 /$ atm-21-1860

View this article at: http://dx.doi.org/10.21037/atm-21-1860

\section{Introduction}

Hilar cholangiocarcinoma, also known as Klatskin tumor, is a malignant tumor that occurs at the confluence of the right and left hepatic bile ducts and accounts for approximately $50-70 \%$ of all cholangiocarcinomas $(1,2)$. Although it is less common in some Western countries, hilar cholangiocarcinoma is one of the most common causes of malignant biliary obstruction in the Asia-Pacific region (3). The prognosis of hilar cholangiocarcinoma is extremely poor, with a 5 -year survival rate of $9-18 \%$ (4). At present, the only effective treatment is radical surgical resection (5).

Bismuth (6) classified hilar cholangiocarcinoma into types I, II, III, and IV according to the extent of tumor infiltration into the biliary tract. For type II or above, effective radical resection tends to involve major liver resection and lymph node dissection (7). However, due to tumors blocking the large bile duct, almost all hilar cholangiocarcinoma patients develop varying degrees of jaundice before surgery (8). Persistently aggravated hyperbilirubinemia severely damages multiple systems in the body (9). Treatment for these diseases, including a series of operations, such as liver resection, lymph node dissection, or anastomosis of the biliary and intestinal tracts, increases the chance of complications. Therefore, the incidence of postoperative complications in patients with hilar cholangiocarcinoma undergoing major liver resection can be as high as $36.5 \%(10)$.

Bismuth classification (11), serum albumin level $(11,12)$, preoperative bilirubin level (10), increased neutrophil and lymphocyte counts (10), future liver remnant (FLR) (12), and other related risk factors have been successively reported to help predict the risk of complications and mortality. However, these approaches are still insufficient in terms of accurately predicting short-term outcomes. Some studies $(13,14)$ have used surgical risk prediction systems for hilar cholangiocarcinoma surgery, including the Physiological and Operative Severity Score for the Enumeration of Mortality and Morbidity (POSSUM), the Portsmouth modification of POSSUM (P-POSSUM), and Estimation of Physiologic Ability and Surgical Stress (E-PASS), and found all of them to be effective. However, these prediction systems have a wide scope of application that cannot fully reflect the specific characteristics of diseases such as hilar cholangiocarcinoma. The accuracy of these systems in reflecting differences in the risks of surgeries with varying degrees of complexity is insufficient. A recent multicenter study (15) proposed a special scoring system for the prediction of mortality risk after major liver resection for hilar cholangiocarcinoma. The study highlighted five key factors: "age", "preoperative cholangitis", "FLR volume $<30 \%$ ", "portal vein reconstruction", and "incomplete FLR drainage in patients with FLR volume $<50 \%$ ", which were used to establish a mortality risk prediction model. However, the discriminability was lower than that of more general scoring systems and only predicted mortality (not postoperative complications), and thus, this model had limited clinical guiding significance.

There is currently no preoperative risk assessment system for predicting complications after radical resection of hilar cholangiocarcinoma. This study aimed to explore factors related to the risk of complications and hepatic failure after radical resection of hilar cholangiocarcinoma. We hypothesized that the damage caused by hyperbilirubinemia would likely be an important factor. The resulting information will support the establishment of a preoperative safety assessment and prediction model for radical resection of Bismuth II or above hilar cholangiocarcinoma. This study will also provide a basis for developing reasonable and safe preoperative strategies by quantifying the degree of damage caused by hyperbilirubinemia. We present the following article in accordance with the STROBE reporting checklist (available at http://dx.doi.org/10.21037/atm-211860).

\section{Methods}

\section{Patients}

This was a retrospective study. Patients who underwent radical resection of hilar cholangiocarcinoma at the Department of Hepatobiliary Surgery, West China Hospital of Sichuan University, from April 2010 to January 2018 were included.

The inclusion criteria were as follows: (I) patients who underwent radical resection of hilar cholangiocarcinoma; (II) patients who underwent $\mathrm{R} 0$ resection with no cancer accumulation of the resection margin under microscope and 
R1 resection with cancer accumulation under microscope (invisible to the naked eye); and (III) patients who experienced preoperative complications with obstructive jaundice. The exclusion criteria were as follows: (I) patients with a hilar cholangiocarcinoma grade below Bismuth type II or type II patients with an unresected liver; (II) patients who experienced preoperative complications with chronic liver disease; (III) patients who required more time for abdominal or intestinal adhesion lysis due to a history of multiple surgeries; (IV) patients who experienced intraoperative bleeding not due to radical factors of hilar cholangiocarcinoma; (V) patients whose postoperative recovery was affected due to being complicated with severe systemic illnesses; (VI) patients who required emergency surgery due to tumor rupture and bleeding; and (VII) patients with incomplete data of key indicators.

This study was approved by the ethics committee of West China Hospital of Sichuan University, and the requirements for informed consent were waived. All procedures performed in this study involving human participants were in accordance with the Declaration of Helsinki (as revised in 2013).

\section{Procedure}

The patients' clinical data were retrospectively analyzed. In particular, the degree of damage caused by hyperbilirubinemia was quantified to establish a preoperative safety assessment and prediction model for the radical resection of Bismuth II or above hilar cholangiocarcinoma.

\section{Preoperative management and surgery}

All patients underwent enhanced computed tomography (CT), magnetic resonance imaging (MRI), and threedimensional (3D) vascular reconstruction to classify their tumors, understand vascular invasion, and to calculate the residual liver volume in order to develop a surgical plan. Jaundice reduction methods included percutaneous transhepatic biliary drainage (PTBD) and endoscopic biliary drainage (ENBD).

All patients underwent radical resection of hilar cholangiocarcinoma. According to the differential classifications of cancer, a variety of procedures, including liver resection with different ranges, resection of the bile duct (including the tumor), Roux-en-y anastomosis, and hepatic hilar lymph node dissection, were performed.
The same team carried out all of the surgeries. The team included dozens of doctoral advisors/professors from the Department of Hepatobiliary Surgery of West China Hospital of Sichuan University. The clinical experience and technical skills of the team members were uniform.

An upper abdominal reverse "L"-shaped incision was made to maximize exposure of the right liver. Intraoperative ultrasonography was conducted to determine the presence or absence of newly found metastatic lesions and the extent of liver resection. The hepatic portal structure was skeletonized, and blood vessels (including hepatic arteries and portal veins) were repaired and reconstructed if necessary. The methods used during liver resection varied according to the preferences of the different surgeons, such as the use of the forceps-clamping method, an ultrasound knife, or cavitron ultrasonic surgical aspirator (CUSA). Due to the different ranges of liver resection and numbers of residual bile duct branches, Roux-en-y anastomosis aimed to minimize the number of anastomotic stomas.

\section{Cumulative damage effect of jaundice (CDEF)}

Studies have shown that the incidence of complications and mortality significantly increases after hepatectomy in patients with obstructive jaundice (2). Therefore, most relevant research has included the preoperative bilirubin level as one of the risk factors for predicting postoperative complications $(7,12)$. However, almost all studies have overlooked one factor: the duration of preoperative jaundice. For example, if jaundice lasts for 1 week or 1 month, the damage caused is clearly different. Of course, at the same time, we cannot ignore the level of serum bilirubin. For this reason, we established a new conceptthe "cumulative damage effect of jaundice"-which is calculated as follows: CDEJ instantaneous value $=$ serum instantaneous bilirubin value $\times$ instantaneous time.

Figure 1 shows that the instantaneous value of CDEJ is the area of the vertical line drawn from each instantaneous point on the jaundice curve to the abscissa. The area under the jaundice curve is defined as the CDEJ in the whole time period from the onset of jaundice until after the reduction of jaundice. However, there is a general consensus in the industry that bilirubin $<2 \mathrm{mg} / \mathrm{dL}$ is invisible jaundice, which will minimally affect the body. Therefore, the calculation method for CDEJ is: [(20 mg/dL $-2 \mathrm{mg} / \mathrm{dL}) / 2] \times \mathrm{dp}+$ [(20 mg/dL $+5 \mathrm{mg} / \mathrm{dL}-4 \mathrm{mg} / \mathrm{dL}) / 2] \times$ do

(dp: days prior to jaundice reduction; do: days of jaundice reduction). 
Page 4 of 12

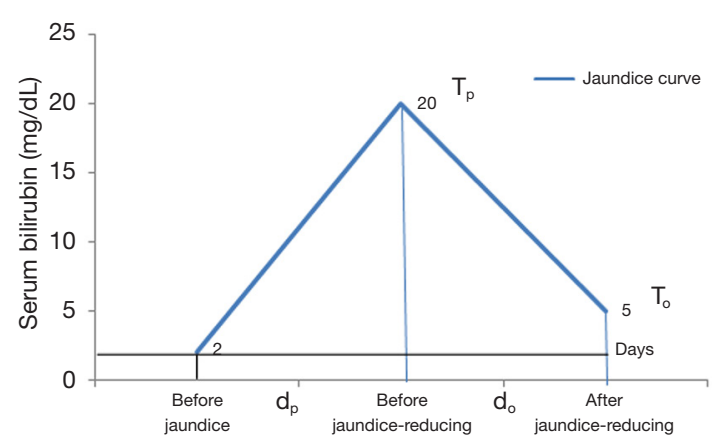

Figure 1 Simulated diagram of changes in jaundice.

This hypothetical situation is the simplest pattern of change in jaundice, which is an approximate value that can only be obtained when the peak bilirubin value and the preoperative value are known. In the real world, the more intensive the preoperative blood biochemical test is, the higher the obtained bilirubin value will be. The measured area value is closer to the true value. Therefore, according to this simplest pattern of change in jaundice, we will obtain the calculation formula: $\mathrm{CDEJ}=[(\mathrm{Tp}-2 \mathrm{mg} / \mathrm{dL}) / 2] \times \mathrm{dp}+$ $[(\mathrm{Tp}+\mathrm{To}-4 \mathrm{mg} / \mathrm{dL}) / 2] \times$ do

( $\mathrm{Tp}$ : the highest preoperative bilirubin value; To: preoperative bilirubin value; dp: days prior to jaundice reduction; do: days of jaundice reduction).

In the formula, $\left[\left(T_{p}-2 \mathrm{mg} / \mathrm{dL}\right) / 2\right] \times \mathrm{d}_{\mathrm{p}}$ represents the CDEJ before jaundice reduction; that is, the triangular area under the curve from the onset of jaundice to before jaundice reduction, as shown in Figure 1. If multiple serum bilirubin values are measured in this time period, this triangle will become to be several trapezoids, and the formula will become the sum areas of these trapezoids; While, in the formular, $\left[\left(T_{p}+T_{0}-4 \mathrm{mg} / \mathrm{dL}\right) / 2\right]$, represents the CDEJ during jaundice reduction; that is, the trapezoidal area under the jaundice curve during the period from the beginning of the reduction in jaundice to the preoperative period. Similarly, once more serum bilirubin values are measured in this time period, the formula will also come to represent the sum of multiple trapezoidal areas.

The details are as follows:

If several serum bilirubin values measured before surgery are: $\mathrm{T}_{1}, \mathrm{~T}_{2}, \mathrm{~T}_{3} \ldots \cdots_{\mathrm{N}}(\mu \mathrm{mol} / \mathrm{L})(1 \mathrm{mg} / \mathrm{dL}=17.1 \mu \mathrm{mol} / \mathrm{L})$.

The distance between each time point of serum bilirubin value determination is $D_{1}, D_{2}, D_{3} \ldots D_{N}$ (days)

The final formula is: $\mathrm{CDEJ}=\left(\mathrm{T}_{1}-34.2\right) \times \mathrm{D} 1+\left[\left(\mathrm{T}_{1}+\mathrm{T}_{2}\right.\right.$ $\left.-68.4) \times \mathrm{D}_{2} / 2\right]+\left[\left(\mathrm{T}_{2}+\mathrm{T}_{3}-68.4\right) \times \mathrm{D}_{3} / 2\right]+\ldots \ldots . .\left[\left(\mathrm{T}_{\mathrm{N}-1}+\mathrm{T}_{\mathrm{N}}\right.\right.$ $\left.-68.4) \times D_{N} / 2\right]$

\section{Luo et al. Complications of hilar cholangiocarcinoma resection}

The effect of the degree of liver reserve function (LRF) recovery after jaundice reduction on the safety of surgery should also be considered. The declining rate of bilirubin after jaundice reduction was used to represent this degree $(\mathrm{LRF})(16): \mathrm{LRF}=\left[\left(\mathrm{T}_{\mathrm{p}}-\mathrm{T}_{\mathrm{o}}\right) / \mathrm{T}_{\mathrm{p}}\right] / \mathrm{d}_{0}$.

\section{Data collection and definitions}

All clinical data were retrieved from the patients' medical records, including medical history, progress notes, surgical records, the outcomes of various tests and examinations, and pathological results. The indicators collected included age, sex, body mass index (BMI), preoperative cholangitis, preoperative bilirubin, preoperative albumin protein, FLR, LRF, postoperative mortality, and CDEJ.

For diagnosis of cholangitis (17), at least two of the following had to be met at the same time: (I) body temperature $>38.5^{\circ} \mathrm{C}$; (II) white blood cells $>12 \times 10^{9} / \mathrm{L}$ or $<4.0 \times 10^{9} / \mathrm{L}$; and (III) right upper abdomen pain present in patients with positive bile cultures. The outcomes were the incidence of complications, hepatic failure, bile leakage, hemorrhage and infection.

The diagnostic criteria for hepatic failure (12) were as follows: serum bilirubin $>7 \mathrm{mg} / \mathrm{dL}$ or more than the preoperative level at least 5 days after surgery. Bile leakage (18) was defined according to the following: on the 3 rd day postoperatively, elevated bilirubin concentration in the abdominal drainage fluid or abdominal cavity fluid, or the need for radiological intervention (i.e., interventional drainage) due to cholestasis or biliary peritonitis. An increase in the bilirubin concentration in the drainage or peritoneal fluid was defined as a bilirubin concentration at least three times the normal amount in the serum measured at the same time.

The diagnostic criteria for hemorrhage (19) were as follows: (I) postoperative hemoglobin value was $3 \mathrm{~g} / \mathrm{dL}$ lower than the postoperative baseline standard (the hemoglobin value was immediately reviewed upon completion of the operation); and/or (II) any postoperative causes for decreased hemoglobin that required red blood cell transfusion and/or invasive re-intervention (such as embolization or re-laparotomy) to stop the bleeding.

Diagnosis of infection: the following manifestations occur on the 3rd day or more after surgery, and three of them must be met simultaneously: (I) body temperature $>38$ or $<36^{\circ} \mathrm{C}$; (II) white blood cell $>12 \times 10^{9} / \mathrm{L}$ or $<4.0 \times 10^{9} / \mathrm{L}$ or immature cells $>10 \%$; (III) symptoms related to the infected site, such as cough and sputum, abdominal pain, frequent or 
painful urination, and incision swelling, etc.; (IV) positive body fluid tests, such as a positive body fluid culture and pus cells, etc.; and (V) imaging that shows signs of infection.

The incidence of complications was defined as any of the above complications.

\section{Follow-up}

The last follow-up was 60 days after surgery. The indicators assessed at follow-up included the incidence of complications and all of the above complications.

\section{Statistical analysis}

SPSS 25.0 (IBM, Armonk, NY, USA) was used for statistical analysis. Continuous data were assessed using the Shapiro-Wilk test for normally distributed data, which were expressed as the means \pm standard deviation (SD). Meanwhile, non-normally distributed continuous data were expressed as medians (Q1, Q3). Categorical data were expressed as n (\%). Normally distributed continuous data were tested using Student's $t$ test or analysis of variance (ANOVA), while non-normally distributed continuous data were analyzed via the Mann-Whitney $U$ test or KruskalWallis test. Categorical data were analyzed by the chisquare test or Fisher's exact test. Variables with $\mathrm{P} \leq 0.05$ in the univariate analysis indicated it was clinically significant, in the multivariate logistic analysis via the enter method. Logistic regression models were established to examine risk factors for five outcomes based on associated factors with significance. $\mathrm{P}<0.05$ was considered statistically significant.

The area under the receiver operating characteristic (ROC) curve (AUC) was used to determine the diagnostic value of the model in predicting postoperative complications and to calculate the optimal cutoff value. The sensitivity, specificity, accuracy, as well as the positive and negative predictive values were calculated based on the optimal cutoff value.

All of the prediction models were presented graphically as nomograms. Nomograms were constructed using multivariable logistic regression models for complications.

\section{Results}

\section{Baseline characteristics}

A total of 171 patients aged $57.8 \pm 10.0$ years (median age, 60 ; range, 30-78) were included in this study. There were more males than females [98 (57.3\%) vs. $73(42.7 \%)$ ]. Thirty-two (18.7\%) patients had combined preoperative cholangitis. The CDEJ was $3,068.75$ (74.8, 30,028.4). Tables 1 and 2 outline the baseline characteristics.

\section{Postoperative complications}

There were 115 (67.3\%) patients with complications. The most common complication was infection, which occurred in $59(34.5 \%)$ patients, followed by hemorrhage in 44 (25.7\%) patients, hepatic failure in $39(22.8 \%)$ patients, and bile leakage in 35 (20.5\%) patients.

\section{Receiver operating curve of complications and hepatic failure}

ROC curve analysis was performed to establish whether the factors identified by our analysis were effective in predicting the incidence of postoperative complications. Using a cutoff value of 2,151 for CDEJ, the resulting AUC was 0.69 (95\% CI $=0.615-0.759)$, with a sensitivity of $66.09 \%$ and a specificity of $69.64 \%$. The optimal cutoff for preoperative bilirubin was $111.7 \mu \mathrm{mol} / \mathrm{L}$, which yielded an AUC of 0.65 (95\% CI $=0.573-0.721)$, a sensitivity of $84.35 \%$, and a specificity of $42.86 \%$ (Figure 2).

Using a similar method, we subsequently analyzed the effectiveness of CDEJ in predicting hepatic failure (Figure 2). When the optimal cutoff was 3931.95 for CDEJ, the AUC was 0.605 (95\% CI $=0.582-0.679)$, the sensitivity was $51.28 \%$, and the specificity was $70.45 \%$. At an optimal cutoff of $115.9 \mu \mathrm{mol} / \mathrm{L}$ for preoperative bilirubin, the AUC was 0.638 (95\% CI $=0.561-0.71)$, the sensitivity was $92.31 \%$, and the specificity was $32.58 \%$. When the optimal cutoff for age was 50 years, the sensitivity was $97.44 \%$, the specificity was $27.27 \%$, and the AUC was 0.64 (95\% $\mathrm{CI}=0.563-0.712)$. When the optimal cutoff for FLR was 50 , the sensitivity was $48.72 \%$, the specificity was $78.79 \%$, and the AUC was 0.638 (95\% CI $=0.515-0.667)$.

\section{Univariate and multivariate factors associated with the incidence of complications and bepatic failure}

Univariate analysis identified that cholangitis $(\mathrm{P}=0.002)$, preoperative bilirubin $(\mathrm{P}=0.002)$, preoperative albumin protein $(\mathrm{P}=0.014)$, and CEDJ $(\mathrm{P}<0.001)$ were associated with the incidence of postoperative complications (Table 1). The factors associated with hepatic failure were age $(\mathrm{P}=0.008)$, preoperative bilirubin $(\mathrm{P}=0.009)$, and CEDJ 
Table 1 Baseline characteristics, univariate analysis between patients with or without complications, and multivariate logistic analysis for the incidence of complications

\begin{tabular}{|c|c|c|c|c|c|c|}
\hline Variable & \multicolumn{3}{|c|}{ Univariate } & \multicolumn{3}{|c|}{ Multivariate } \\
\hline Age, median (Q1, Q3), year & $60(50.3-63)$ & $59(53-64)$ & 0.479 & & & \\
\hline Gender, n (\%) & & & 0.719 & & & \\
\hline Male & $31(55.4)$ & $67(58.3)$ & & & & \\
\hline BMI, mean \pm SD & $21.54 \pm 2.37$ & $22.28 \pm 2.52$ & 0.069 & & & \\
\hline Cholangitis, n (\%) & $3(5.4)$ & $29(25.2)$ & 0.002 & 9.638 & $2.683-34.622$ & 0.001 \\
\hline Preoperative bilirubin, median (Q1, Q3) & $156.25(80.03-223.2)$ & $194.2(141.7-278.3)$ & 0.002 & 1.006 & $1.002-1.01$ & 0.004 \\
\hline $\begin{array}{l}\text { Preoperative albumin protein, median } \\
\text { (Q1, Q3) }\end{array}$ & $37.85(35.68-40.38)$ & $36.4(33.9-39.1)$ & 0.014 & 0.949 & $0.866-1.039$ & 0.257 \\
\hline CDEJ, median (Q1, Q3) & $1,587.58(709.34-3,108.9)$ & $3,057.3(1,563.6-5,767.1)$ & $<0.001$ & 1.0001 & $1.000027-1.000239$ & 0.014 \\
\hline
\end{tabular}

Table 2 Univariate and multivariate analysis for hepatic failure

\begin{tabular}{|c|c|c|c|c|c|c|}
\hline Variable & \multicolumn{3}{|c|}{ Univariate } & \multicolumn{3}{|c|}{ Multivariate } \\
\hline Age, median (Q1, Q3), year & $59(50-63)$ & $63(58-65)$ & 0.008 & 1.083 & $1.029-1.14$ & 0.002 \\
\hline Gender, n (\%) & & & 0.329 & & & \\
\hline Male & $73(55.3)$ & $25(64.1)$ & & & & \\
\hline $\mathrm{BMI}$, mean $\pm \mathrm{SD}$ & $21.88 \pm 2.44$ & $22.59 \pm 2.62$ & 0.117 & & & \\
\hline Cholangitis, n (\%) & $22(16.7)$ & $10(25.6)$ & 0.207 & & & \\
\hline Preoperative bilirubin, median (Q1, Q3) & $172.65(101.13-258.05)$ & $235.5(160.3-311.3)$ & 0.009 & 1.008 & $1.004-1.012$ & $<0.001$ \\
\hline $\begin{array}{l}\text { Preoperative albumin protein, median } \\
\text { (Q1, Q3) }\end{array}$ & $37.3(34.38-39.6)$ & $36.7(34-39.1)$ & 0.504 & & & \\
\hline CDEJ, median (Q1, Q3) & $2,260(1,320.13-4,937.64)$ & $3,944.9(1,548.3-7,061.5)$ & 0.046 & 1.0001 & $1.00001-1.00019$ & 0.024 \\
\hline
\end{tabular}

$\mathrm{P}<0.05$ indicated statistic significant. *, although the $\mathrm{P}>0.05$, considering its great clinical significance, the single-factor Logistic test was performed and with $\mathrm{P}<0.05$.BMI, body mass index; FLR, future liver remnant; LRF, liver reserve function; CDEJ, cumulative damage effect of jaundice. 

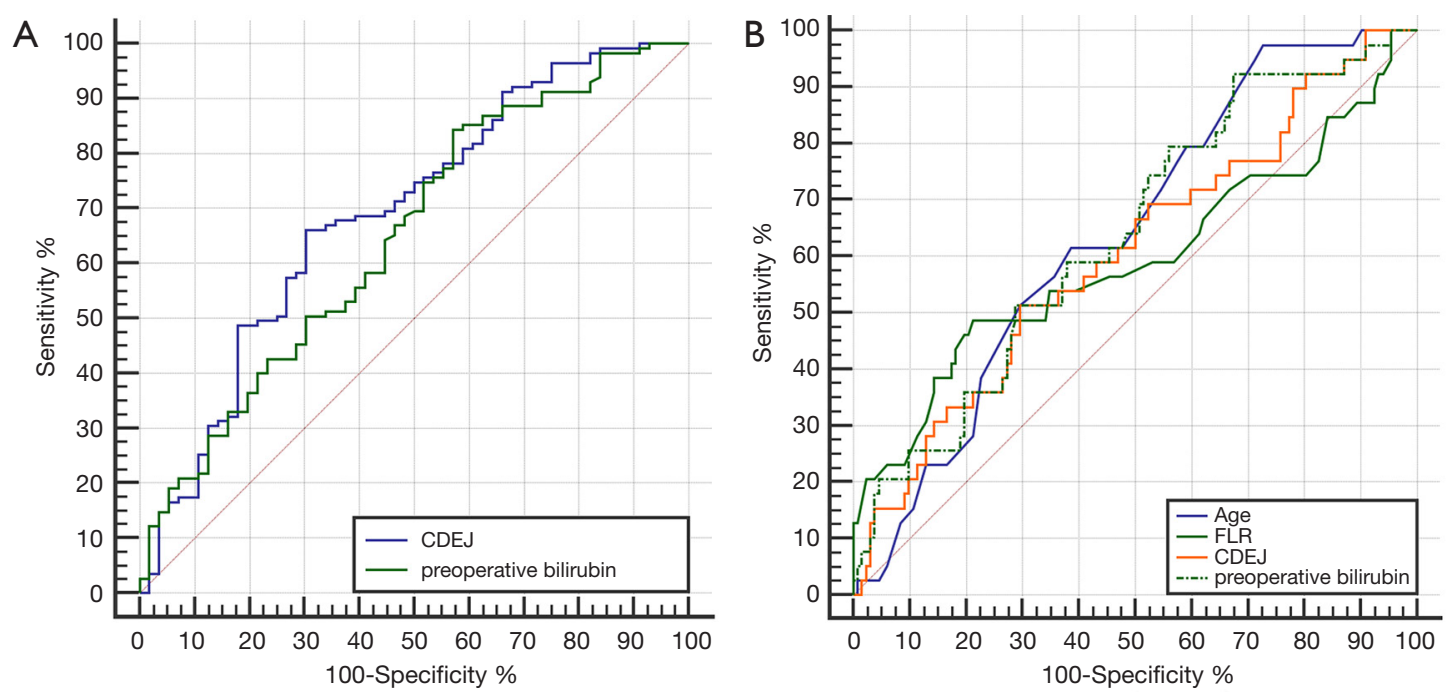

Figure 2 Receiver operating characteristic (ROC) curve predicting the incidence of complications and hepatic failure. (A) ROC curve for incidence of complications; when the optimal cutoff was 2,151 for the cumulative damage effect of jaundice (CDEJ), the sensitivity was $66.09 \%$, the specificity was $69.64 \%$, and the area under the curve (AUC) was 0.69 (95\% CI $=0.615-0.759$ ). When the optimal cutoff for preoperative bilirubin was $111.7 \mu \mathrm{mol} / \mathrm{L}$, the sensitivity was $84.35 \%$, the specificity was $42.86 \%$, and the AUC was 0.65 (95\% CI $=0.573-$ 0.721). (B) ROC curve for hepatic failure. When the optimal cutoff was 3,931.95 for CDEJ, the sensitivity was $51.28 \%$, the specificity was $70.45 \%$, and the AUC was 0.605 (95\% CI =0.582-0.679). When the optimal cutoff for preoperative bilirubin was $115.9 \mu m o l / L$, the sensitivity was $92.31 \%$, the specificity was $32.58 \%$, and AUC was 0.638 (95\% CI =0.561-0.71). When the optimal cutoff for age was 50 years, the sensitivity was $97.44 \%$, the specificity was $27.27 \%$, and AUC was 0.64 (95\% CI $=0.563-0.712$ ). When the optimal cutoff for FLR was 50 , the sensitivity was $48.72 \%$, the specificity was $78.79 \%$, and AUC was 0.638 (95\% CI =0.515-0.667).

\section{( $\mathrm{P}=0.046)$ (Table 2).}

Multivariate analysis determined that cholangitis ( $\mathrm{OR}=9.638,95 \% \mathrm{CI}=2.683-34.622, \mathrm{P}=0.01)$, preoperative bilirubin $(\mathrm{OR}=1.006,95 \% \mathrm{CI}=1.002-1.01, \mathrm{P}=0.04)$, and CDEJ $(\mathrm{OR}=1.0001,95 \% \mathrm{CI}=1.000027-1.000239$, $\mathrm{P}=0.014$ ) were significant independent factors associated with the occurrence of complications (Table 1). It also found that age $(\mathrm{OR}=1.083,95 \% \mathrm{CI}=1.029-1.14, \mathrm{P}=0.002)$, preoperative bilirubin $(\mathrm{OR}=1.008,95 \% \mathrm{CI}=1.004-1.012$, $\mathrm{P}<0.001)$, FLR $(\mathrm{OR}=0.963,95 \% \mathrm{CI}=0.941-0.986, \mathrm{P}=0.02)$, and $\mathrm{CDEJ}(\mathrm{OR}=1.0001,95 \% \mathrm{CI}=1.00001-1.00019$, $\mathrm{P}=0.024)$ were independently associated with hepatic failure (Table 2). Furthermore, CDEJ was not associated with bile leakage $(\mathrm{P}=0.498)$, hemorrhage $(\mathrm{P}=0.068)$, or infection $(\mathrm{P}=0.05)$ (Tables $\mathrm{S} 1-\mathrm{S} 3)$.

\section{Establishment of the nomogram for predicting the incidence of complications and hepatic failure}

Based on the multivariate analysis, a nomogram for the incidence of complications incorporating three independent factors was constructed (Figure $3 A$ ). The calibration curve of the nomogram showed good calibration (Figure $3 B$ ). The prediction accuracy of the concordance index(c-index) was $80.04 \%$ (95\% CI $=78.23-81.91 \%$ ), and another nomogram for the hepatic failure incorporating four independent factors was constructed (Figure $4 A$ ). The calibration curve of the nomogram showed good calibration (Figure 4B). The prediction accuracy of the c-index was $80.75 \%$ ( $95 \%$ $\mathrm{CI}=77.33-82.51 \%)$.

\section{Discussion}

The aim of this study was to assess the association between CDEJ and the incidence of complications, especially hepatic failure, after surgical resection of hilar cholangiocarcinoma (graded at Bismuth II or above). The results showed that CDEJ, cholangitis, and preoperative bilirubin were independently associated with the incidence of complications. In addition, age, CDEJ, preoperative bilirubin, and FLR were independently associated with hepatic failure. When CDEJ was used to predict the 


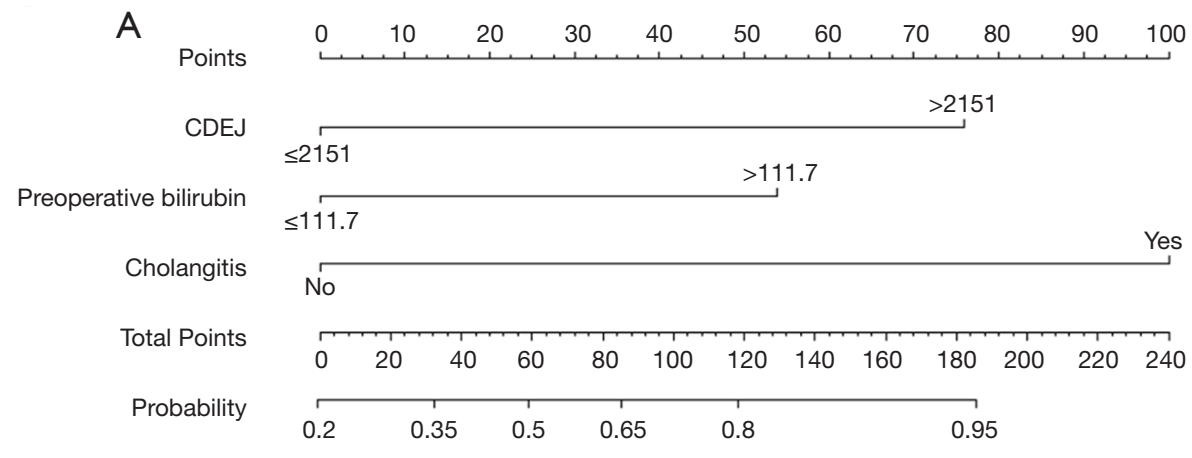

B

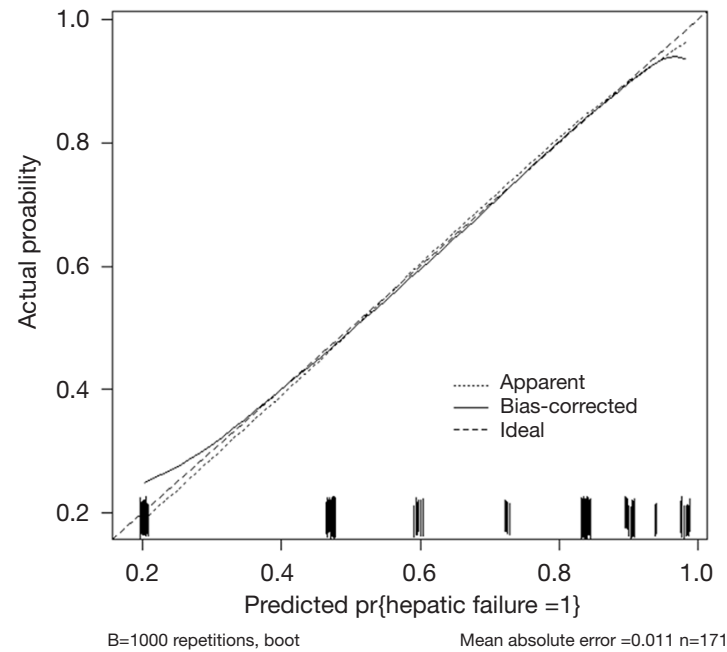

Figure 3 Nomogram for predicting incidence of complications and calibration plot. (A) The nomogram maps the predicted probability of the incidence of complications on a scale of 0 to 240 . With $50 \%$ as the cutoff point, a score of greater than 59 points indicates that the patient will have complications. The prediction accuracy (c-index) of the above scoring system is $80.04 \%(95 \% \mathrm{CI}=78.23-81.91 \%)$. (B) Calibration curves of the nomogram.

incidence of complications, the resulting AUC was 0.69 with a sensitivity of $66.09 \%$ and specificity of $69.64 \%$. For hepatic failure prediction, the CDEJ had an AUC of 0.605 , a sensitivity of $51.28 \%$, and a specificity of $70.45 \%$. The calibrations of the two nomograms for predicting the incidence of complications and hepatic failure were good.

In this study, the rate of postoperative complications was $67.3 \%$. This rate is higher than the approximate morbidity rate $(39 \%)$ found in a summary of 12 different studies. However, these findings included patients with Bismuth type I carcinomas with lower complication rates, ranging from 0 to $100 \%$ (20). The patients in the present study should be considered to be at higher risk for postoperative complications, as they were all Bismuth type II or above. The mean age of the patients $(57.8 \pm 10.0$ years) was slightly younger than that previously described in the USA, where the ages of patients with hilar cholangiocarcinoma typically range between 60 and 70 years old (21). However, globally, cholangiocarcinoma is usually diagnosed in individuals aged over 50 years (on average) (22).

The previous prediction model for mortality after surgical resection for hilar cholangiocarcinoma identified risk factors including age, preoperative cholangitis, FLR volume $<30 \%$, portal vein reconstruction, and incomplete FLR drainage in patients with FLR volume $<50 \%$ (15). Although the current study was focused more on postoperative complications than on mortality, preoperative cholangitis was independently associated with the incidence of complications. Therefore, our results are consistent with the previous research to a certain extent. However, CDEJ was also independently associated with the incidence of complications, highlighting the importance of jaundice- 


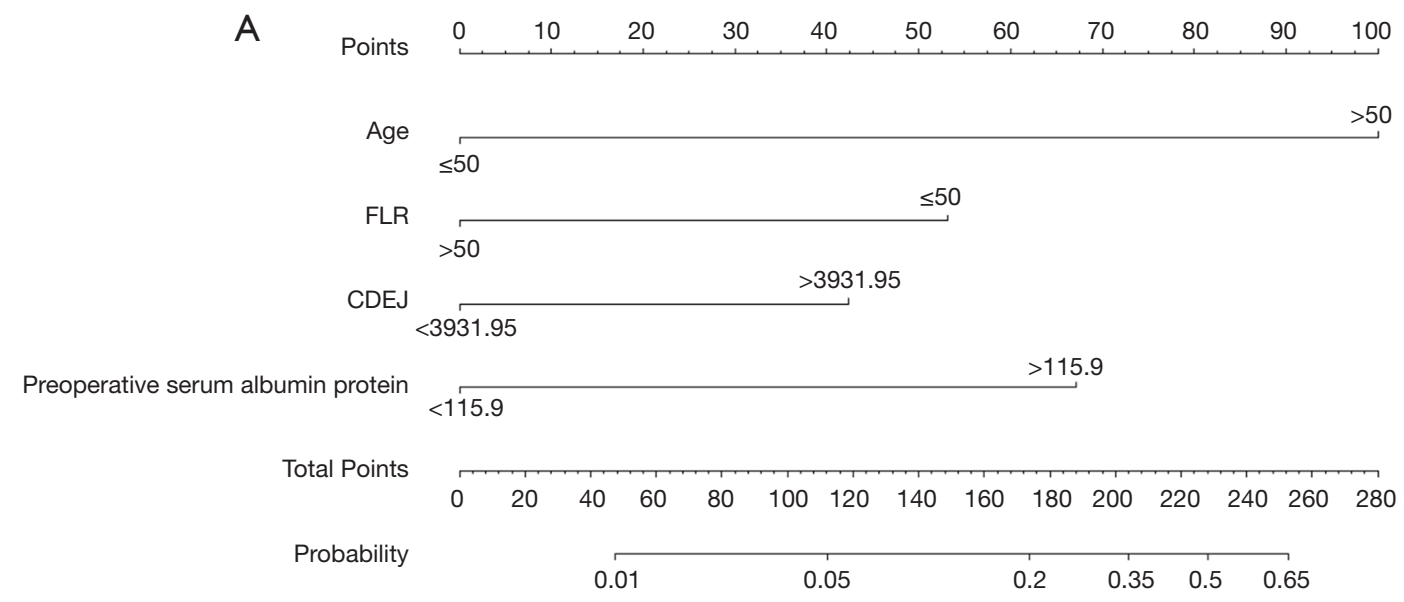

B

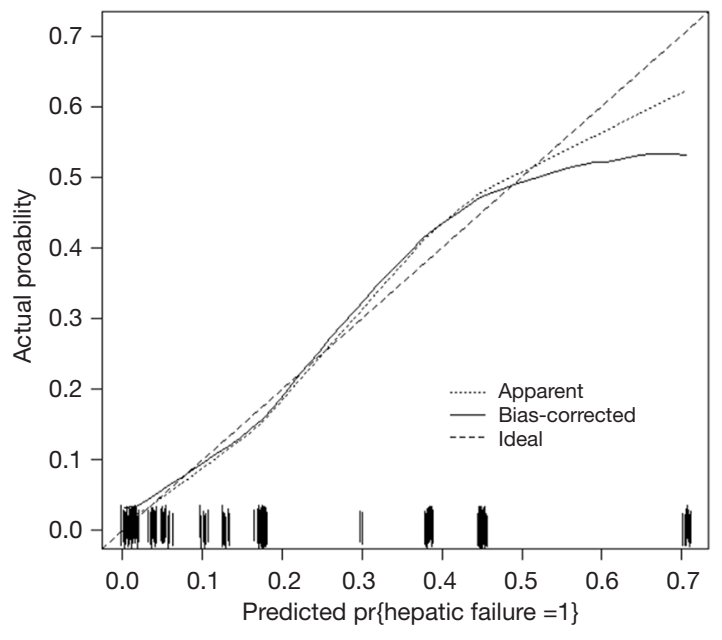

Figure 4 Nomogram for predicting hepatic failure and calibration plot. (A) The nomogram maps the predicted probability of hepatic failure on a scale of 0 to 280 . With $50 \%$ as the cutoff point, a score of greater than 228 points indicates that the patient will have hepatic failure. The prediction accuracy (c-index) of the above scoring system is $80.75 \%$ (95\% CI $=77.33-82.51 \%$ ). (B) Calibration curves of the nomogram.

induced damage.

Jaundice damage is fairly well established as an important factor related to the outcomes of patients with hilar cholangiocarcinoma. The morbidity and mortality rates associated with liver resection are significantly higher in patients with obstructive jaundice than in patients with normal liver function (23). Jaundice leads to systemic inflammation, which then causes multiple organ dysfunction, kills or damages hepatocytes and Kupffer cells, and markedly lowers the number of natural killer $\mathrm{T}$ cells in the liver. These phenomena result in impaired hepatic and systemic immune function (24). In this way, jaundice is implicated in a high number of complications in patients with hilar cholangiocarcinoma. This has led to some debate over the benefits of biliary drainage before and after surgery, which, while relieving jaundice, may increase the risk of complications due to an increased number of procedures (25). Most studies suggest some form of preoperative drainage of the FLR for most jaundiced patients with hilar cholangiocarcinoma (26). A rat model of obstructive jaundice clearly showed that oxidative damage occurs in the liver and kidneys with jaundice, and that prolonged jaundice increases the damage, eventually making it irreversible (27).

This knowledge led to our development of a method to calculate the cumulative damage due to jaundice that takes into account the time that the patient has been experiencing 
jaundice. The importance of CDEJ in this study indicates that jaundice may have a cumulative damaging effect to multiple systems throughout the body over time. In obstructive jaundice, persistent (or even worsening) jaundice results in the continuous release of inflammatory factors, causing cumulative damage to various systems. Even if jaundice is reduced, the body might not be able to adequately remove these inflammatory factors if a sufficient amount of time elapses. Cholangitis was also identified as being independently associated with the incidence of complications in the present study. Cholangitis can also increase the release of inflammatory factors, triggering systemic inflammatory response syndrome, and coupled with surgical trauma stress, the body is unable to repeatedly clear inflammatory factors, ultimately leading to slower healing and complications.

In this study, CDEJ, age, FLR, and preoperative bilirubin were also found to be independently associated with hepatic failure. In addition to the reasons discussed above, these associations may be related to high bile acidinduced hepatocyte apoptosis due to persistent obstructive jaundice (28-31). We further explored the value of CDEJ in predicting complications and hepatic failure, and found that CDEJ predicted complications with an AUC of 0.69 , a sensitivity of $66.09 \%$, and a specificity of $69.64 \%$. Using preoperative bilirubin to predict complications yielded an AUC of 0.65 , with a sensitivity of $84.35 \%$ and a specificity of $42.86 \%$. Predicting hepatic failure with cumulative damage due to CDEJ had an AUC of 0.605 , with a sensitivity of $51.28 \%$ and a specificity of $70.45 \%$, while preoperative bilirubin had an AUC of 0.638 , with a sensitivity of $92.31 \%$ and a specificity of $32.58 \%$.

The preoperative serum bilirubin level is mainly improved through preoperative jaundice-reducing measures. However, long-term disregard of the effect of jaundice reduction will increase CDEJ, which may heighten the risk of postoperative complications. Therefore, it may be necessary to repeatedly consider the influence of the preoperative bilirubin level and CDEJ on the risk of postoperative complications. This also demonstrates the significance of this study for clinical translation. Overall, these findings suggest that CDEJ is reasonably useful in predicting complications. However, the AUCs for each measure were lower than those of a previous investigation, which found that the POSSUM system predicted morbidity risk with an AUC of 0.843 (14). Also, the perihilar cholangiocarcinoma risk score for mortality after surgical resection was achieved, and the AUC was 0.75 (15), which was slightly lower than that of CDEJ in the present study. In part, the low AUCs in the current study may be due to the small number of cases. Generally, the establishment of a risk scoring system is supported by a large sample of multicenter patients. Since hilar cholangiocarcinoma is a rare disease and some patients have been excluded, the number of patients included in this study was limited. However, this study does highlight an original innovation and idea that might be developed further. CDEJ can be calculated fairly easily with known clinical parameters, and the resulting information has some value in predicting complications after resection of hilar cholangiocarcinoma.

The nomogram established in this study shows whether complications are likely to occur. A score $>59$ points indicates that a patient has a $>50 \%$ chance of complications. The prediction accuracy is relatively high, with a c-index of $80 \%$. Among the three factors included in the nomogram, CDEJ and cholangitis alone achieved scores $>59$. This finding reminds us that controlling CDEJ and actively preventing the occurrence of cholangitis should be enforced by increasing the monitoring frequency of serum bilirubin, using CDEJ as the main indicator of close monitoring, operating as early as possible, preventively using antibiotics and anti-inflammatory drugs, and avoiding cholangitis. If jaundice needs to be reduced, the resulting increase in CDEJ and the consequences of cholangitis caused by catheter-related infections must be considered. After careful consideration, the target level of preoperative bilirubin and the time to reduce yellowness can be determined. The nomogram, with liver failure as the outcome, shows that patients with a score $>228$ are at $>50 \%$ risk of liver failure, with a high prediction accuracy (c-index of $81 \%$ ). Regarding the patients' ages, most patients with hilar cholangiocarcinoma are $>50$ years of age, although this is a non-modifiable factor. Therefore, the score can only be reduced through FLR, CDEJ, and preoperative serum albumin levels. In addition, if the FLR can be maintained above $50 \%$, the risk of liver failure can be significantly reduced. This highlights that in the preoperative liver resection planning, the residual liver volume should be preserved as much as possible.

This study has some limitations that should be noted. As a retrospective analysis, some bias may have been introduced in the selection of the patients and data analysis. The model will require testing and validation in prospective studies. Although the number of patients was relatively small, this was a pioneering study, and even if some positive trends can be obtained, it provides valuable information. 
The findings can be used to establish a theoretical basis for a prospective, multicenter, large sample study.

The outcomes of this analysis in terms of the factors associated with complications after resection for hilar cholangiocarcinoma to help establish a preoperative risk prediction model that highlights the importance of jaundice. CDEJ was independently associated with complications and can moderately predict the chance of complications and hepatic failure after surgical resection of hilar cholangiocarcinoma.

\section{Acknowledgments}

The authors would like to thank Associate Professor He Xia from the Statistics Department of University of Electronic Science and Technology of China for helping with the statistical analysis. We would also like to thank Dr. Chen Xing from West China Hospital of Sichuan University for his support in data collection.

Funding: This work was supported by grants from the National Key Technologies R\&D Program (2018YFC1106800), the Natural Science Foundation of China (82070644, 82002572, 82002967, 81972747, 81872004, 81800564, 81770615, 81700555 and 81672882), the Science and Technology Support Program of Sichuan Province (2019YFQ0001), the Science and Technology Program of Tibet Autonomous Region (XZ201801-GB-02), the fellowship of China National Postdoctoral Program for Innative Talents (BX20200225, BX20200227), the China Postdoctoral Science Foundation (2020M673231), the 1.3.5 project for disciplines of excellence, West China Hospital, Sichuan University (ZYJC18008), and the Post-Doctor Research Project, West China Hospital, Sichuan University (20HXBH007).

\section{Footnote}

Reporting Checklist: The authors have completed the STROBE reporting checklist. Available at http://dx.doi. org/10.21037/atm-21-1860

Data Sharing Statement: Available at http://dx.doi. org/10.21037/atm-21-1860

Conflicts of Interest: All authors have completed the ICMJE uniform disclosure form (available at http://dx.doi. org/10.21037/atm-21-1860). The authors have no conflicts of interest to declare.
Ethical Statement: The authors are accountable for all aspects of the work in ensuring that questions related to the accuracy or integrity of any part of the work are appropriately investigated and resolved. This study was approved by the ethics committee of West China Hospital of Sichuan University, and the requirements for informed consent were waived. All procedures performed in this study involving human participants were in accordance with the Declaration of Helsinki (as revised in 2013).

Open Access Statement: This is an Open Access article distributed in accordance with the Creative Commons Attribution-NonCommercial-NoDerivs 4.0 International License (CC BY-NC-ND 4.0), which permits the noncommercial replication and distribution of the article with the strict proviso that no changes or edits are made and the original work is properly cited (including links to both the formal publication through the relevant DOI and the license). See: https://creativecommons.org/licenses/by-nc-nd/4.0/.

\section{References}

1. Lewis HL, Rahnemai-Azar AA, Dillhoff M, et al. Current Management of Perihilar Cholangiocarcinoma and Future Perspectives. Chirurgia (Bucur) 2017;112:193-207.

2. Poruk KE, Pawlik TM, Weiss MJ. Perioperative Management of Hilar Cholangiocarcinoma. J Gastrointest Surg 2015;19:1889-99.

3. Rerknimitr R, Angsuwatcharakon P, Ratanachuek T, et al. Asia-Pacific consensus recommendations for endoscopic and interventional management of hilar cholangiocarcinoma. J Gastroenterol Hepatol 2013;28:593-607.

4. Ni QH, Chen T, Wang J. Classification, staging, and resectability assessment for hilar cholangiocarcinoma. Chin J Hepatobiliary Surg 2013;19:477-80.

5. Capobianco I, Rolinger J, Nadalin S. Resection for Klatskin tumors: technical complexities and results. Transl Gastroenterol Hepatol 2018;3:69.

6. Bismuth $\mathrm{H}$, Corlette MB. Intrahepatic cholangioenteric anastomosis in carcinoma of the hilus of the liver.Surg Gynecol Obstet 1975;140:170-8.

7. Chinese Anti-Cancer Association. Expert consensus on standardized diagnosis and treatment of hilar cholangiocarcinoma (2015). Chin J Hepatobiliary Surg 2015;21:505-11.

8. Hameed A, Pang T, Chiou J, et al. Percutaneous vs. endoscopic pre-operative biliary drainage in hilar 


\section{Page 12 of 12}

cholangiocarcinoma - a systematic review and metaanalysis. HPB (Oxford) 2016;18:400-10.

9. Moole H, Bechtold M, Puli SR. Efficacy of preoperative biliary drainage in malignant obstructive jaundice: a meta-analysis and systematic review. World J Surg Oncol 2016;14:182.

10. Dumitrascu T, Brasoveanu V, Stroescu C, et al. Major hepatectomies for perihilar cholangiocarcinoma: Predictors for clinically relevant postoperative complications using the International Study Group of Liver Surgery definitions. Asian J Surg 2016;39:81-9.

11. Gerhards MF, van Gulik TM, de Wit LT, et al. Evaluation of morbidity and mortality after resection for hilar cholangiocarcinoma--a single center experience. Surgery 2000;127:395-404.

12. Ribero D, Zimmitti G, Aloia TA, et al. Preoperative Cholangitis and Future Liver Remnant Volume Determine the Risk of Liver Failure in Patients Undergoing Resection for Hilar Cholangiocarcinoma. J Am Coll Surg 2016;223:87-97.

13. Haga Y, Miyamoto A, Wada Y, et al. Value of E-PASS models for predicting postoperative morbidity and mortality in resection of perihilar cholangiocarcinoma and gallbladder carcinoma. HPB (Oxford) 2016;18:271-8.

14. Wang H, Wang H, Chen T, et al. Evaluation of the POSSUM, P-POSSUM and E-PASS scores in the surgical treatment of hilar cholangiocarcinoma. World J Surg Oncol 2014;12:191.

15. Wiggers JK, Groot Koerkamp B, Cieslak KP, et al. Postoperative Mortality after Liver Resection for Perihilar Cholangiocarcinoma: Development of a Risk Score and Importance of Biliary Drainage of the Future Liver Remnant. J Am Coll Surg 2016;223:321-331.e1.

16. Tian FZ, Shi L, Tang LJ, et al. Recognition about preoperative jaundice-reducing in the patients with malignant obstructive jaundice (clinical analysis of 28 cases). Chin J Curr Adv Gen Surg 2010;13:1-4.

17. Kiriyama S, Takada T, Strasberg SM, et al. TG13 guidelines for diagnosis and severity grading of acute cholangitis (with videos). J Hepatobiliary Pancreat Sci 2013;20:24-34.

18. Koch M, Garden OJ, Padbury R, et al. Bile leakage after hepatobiliary and pancreatic surgery: a definition and grading of severity by the International Study Group of Liver Surgery. Surgery 2011;149:680-8.

19. Rahbari NN, Garden OJ, Padbury R, et al. Posthepatectomy haemorrhage: a definition and grading by the International Study Group of Liver Surgery (ISGLS).

\section{Luo et al. Complications of hilar cholangiocarcinoma resection}

HPB (Oxford) 2011;13:528-35.

20. $\mathrm{Hu} \mathrm{HJ}, \mathrm{Wu} \mathrm{ZR}$, Jin YW, et al. Minimally invasive surgery for hilar cholangiocarcinoma: state of art and future perspectives. ANZ J Surg 2019;89:476-80.

21. Saha SK, Zhu AX, Fuchs CS, B et al. Forty-Year Trends in Cholangiocarcinoma Incidence in the U.S.: Intrahepatic Disease on the Rise. The oncologist 2016;21:594-9.

22. Blechacz B. Cholangiocarcinoma: Current Knowledge and New Developments. Gut Liver 2017;11:13-26.

23. Belghiti J, Hiramatsu K, Benoist S, et al. Seven hundred forty-seven hepatectomies in the 1990s: an update to evaluate the actual risk of liver resection. J Am Coll Surg 2000;191:38-46.

24. Yang $\mathrm{R}$, Zhu S, Pischke SE, et al. Bile and circulating HMGB1 contributes to systemic inflammation in obstructive jaundice. J Surg Res 2018;228:14-9.

25. Enomoto LM, Dixon MEB, Burdette A, et al. Biliary Drainage Before and After Liver Resection for Perihilar Cholangiocarcinoma. Am Surg 2020;86:628-34.

26. Duan F, Cui L, Bai Y, et al. Comparison of efficacy and complications of endoscopic and percutaneous biliary drainage in malignant obstructive jaundice: a systematic review and meta-analysis. Cancer Imaging 2017;17:27.

27. Ozozan OV, Dinc T, Vural V, et al. An electron microscopy study of liver and kidney damage in an experimental model of obstructive jaundice. Ann Ital Chir 2020;91:122-30.

28. Maillette de Buy Wenniger L, Beuers U. Bile salts and cholestasis. Digestive and Liver Disease 2010;42:409-18.

29. Reinehr R, Graf D, Häussinger D. Bile salt-induced hepatocyte apoptosis involves epidermal growth factor receptor-dependent CD95 tyrosine phosphorylation. Gastroenterology 2003;125:839-53.

30. Reinehr R, Becker S, Keitel V, et al. Bile salt-induced apoptosis involves NADPH oxidase isoform activation. Gastroenterology 2005;129:2009-31.

31. Rust C, Wild N, Bernt C, et al. Bile acid-induced apoptosis in hepatocytes is caspase-6-dependent. J Biol Chem 2009;284:2908-16.

(English Language Editor: A. Kassem)

Cite this article as: Luo L, Yao Y, Liao H, Huang J, Liao M, Wang J, Yuan K, Zeng Y. Cumulative damage effect of jaundice may be an effective predictor of complications in patients undergoing radical resection of Bismuth type II or above hilar cholangiocarcinoma. Ann Transl Med 2021;9(10):861. doi: 10.21037/atm-21-1860 


\section{Supplementary}

Table S1 Univariate and multivariate analysis for bile leakage

\begin{tabular}{|c|c|c|c|c|c|c|}
\hline Variable & \multicolumn{3}{|c|}{ Univariate } & \multicolumn{3}{|c|}{ Multivariate } \\
\hline Age, median (Q1, Q3), year & $60(52-65)$ & $59(54-63)$ & 0.282 & & & \\
\hline Gender, n (\%) & & & 0.058 & & & \\
\hline Male & $73(53.7)$ & $25(71.4)$ & & & & \\
\hline $\mathrm{BMI}$, mean $\pm \mathrm{SD}$ & $22 \pm 2.52$ & $22.18 \pm 2.42$ & 0.706 & & & \\
\hline Cholangitis, n (\%) & $17(12.5)$ & $15(42.9)$ & $<0.001$ & 5.25 & $2.265-12.167$ & $<0.001$ \\
\hline Preoperative bilirubin, median (Q1, Q3) & $177.3(103.5-258.05)$ & $181.5(152.9-297.5)$ & 0.123 & & & \\
\hline $\begin{array}{l}\text { Preoperative albumin protein, median } \\
\text { (Q1, Q3) }\end{array}$ & $37.25(35.1-39.58)$ & $35.7(32.2-39.2)$ & 0.112 & & & \\
\hline CDEJ, median (Q1, Q3) & $2,301.53(1,310.79-5,070.23)$ & $2807.2(1,431.75-5,445.35)$ & 0.498 & & & \\
\hline
\end{tabular}

BMI, body mass index; FLR, future liver remnant; LRF, liver reserve function; CDEJ, cumulative damage effect of jaundice.

Table S2 Univariate and multivariate analysis for hemorrhage

\begin{tabular}{|c|c|c|c|c|c|c|}
\hline Variable & \multicolumn{3}{|c|}{ Univariate } & \multicolumn{3}{|c|}{ Multivariate } \\
\hline Age, median (Q1, Q3), year & $60(54-64)$ & $58.5(51-64)$ & 0.558 & & & \\
\hline Gender, n (\%) & & & 0.667 & & & \\
\hline Male & $74(58.3)$ & $24(54.5)$ & & & & \\
\hline $\mathrm{BMI}$, mean $\pm \mathrm{SD}$ & $22.02 \pm 2.53$ & $22.09 \pm 2.4$ & 0.873 & & & \\
\hline Cholangitis, $\mathrm{n}(\%)$ & $23(18.1)$ & $9(20.5)$ & 0.731 & & & \\
\hline Preoperative bilirubin, median (Q1, Q3) & $171.2(108.2-254.9)$ & $225.7(132.5-303.78)$ & 0.025 & 1.004 & $1.001-1.007$ & 0.014 \\
\hline $\begin{array}{l}\text { Preoperative albumin protein, median } \\
\text { (Q1, Q3) }\end{array}$ & $37.4(34.6-40)$ & $36.15(34.05-38.78)$ & 0.183 & & & \\
\hline CDEJ, median (Q1, Q3) & $2,490.7(1,177.25-4,997.2)$ & $2,645.93(1,692.94-6,304.13)$ & 0.068 & & & \\
\hline
\end{tabular}

BMI, body mass index; FLR, future liver remnant; LRF, liver reserve function; CDEJ, cumulative damage effect of jaundice. 
Table S3 Univariate and multivariate analysis for infection

\begin{tabular}{|c|c|c|c|c|c|c|}
\hline Variable & \multicolumn{3}{|c|}{ Univariate } & \multicolumn{3}{|c|}{ Multivariate } \\
\hline Age, median (Q1, Q3), year & $60(52-64)$ & $59(54-63)$ & 0.996 & & & \\
\hline Gender, n (\%) & & & 0.951 & & & \\
\hline Male & $64(57.1)$ & $34(57.6)$ & & & & \\
\hline $\mathrm{BMI}$, mean $\pm \mathrm{SD}$ & $60(52-64)$ & $59(54-63)$ & 0.996 & & & \\
\hline Cholangitis, n (\%) & $14(12.5)$ & $18(30.5)$ & 0.004 & 3.918 & $1.707-8.997$ & 0.001 \\
\hline Preoperative bilirubin, median (Q1, Q3) & $175.45(99.5-273.75)$ & $184(147.7-258.1)$ & 0.221 & & & \\
\hline $\begin{array}{l}\text { Preoperative albumin protein, median } \\
\text { (Q1, Q3) }\end{array}$ & $37.15(34.38-39.58)$ & $36.7(34-39.5)$ & 0.847 & & & \\
\hline CDEJ, median (Q1, Q3) & $2,069.5(1,230.68-4,570.21)$ & $3,057.3(1,548.3-5,627.65)$ & 0.05 & 1 & $1.0-1.0$ & 0.314 \\
\hline
\end{tabular}

BMI, body mass index; FLR, future liver remnant; LRF, liver reserve function; CDEJ, cumulative damage effect of jaundice. 